\title{
Las tomas de tierra en el origen y desarrollo del Movimiento de Trabajadores Desocupados del Chaco*
}

\author{
Maximiliano Román**
}

\section{Resumen}

El Movimiento de Trabajadores Desocupados, en cuanto fenómeno reciente en la sociedad argentina, se vincula de diversas maneras con las formas precedentes de lucha popular. Una de ellas es la "toma" de tierras fiscales o privadas con el objetivo de construir viviendas o emprendimientos productivos. El presente trabajo pretende esclarecer el proceso de génesis y desarrollo de tres organizaciones pertenecientes al Movimiento de Trabajadores Desocupados de la provincia del Chaco y su relación con las tomas de tierra: en primera instancia, como antecedentes de organización territorial que derivaron en la conformación de agrupaciones específicamente "piqueteras", y posteriormente, como acciones puntuales impulsadas con objetivos preestablecidos por grupos ya constituidos como "piqueteros".

\section{Palabras clave}

Movimiento de Trabajadores Desocupados - toma de tierras piqueteros - lucha de clases.

\footnotetext{
*Artículo recibido 11 de abril de 2012. Aceptado 10 de diciembre de 2012.

* * Profesor en Filosofía - Universidad Nacional del Nordeste, Doctorando en Filosofía UNNE -CONICET, Argentina.
} 


\section{Abstract}

The unemployed workers movement, a recent phenomenon in argentine society, has multiple relations with earlier forms of popular struggle. One of these is land occupation, both private and public areas, in order to build housing or productive projects.

This paper aims to clarify the genesis and development of three organizations within the Movement of Unemployed Workers in the province of Chaco and its relationship with land occupations: firstly, as a precedent of territorial organization that led in forming groups specifically "piqueteras"; and after, as specific actions with predetermined targets driven by established groups as "piqueteros".

\section{Keywords}

Unemployed workers movement - land occupation - piqueteros, class struggle. 


\section{Introducción}

El amplio desarrollo de los estudios sobre movimientos sociales en la historia argentina reciente colocó en el centro del debate teórico el problema de la relación entre "nuevas" y "viejas" formas de lucha popular. Frente al dilema, no pocos autores han recurrido a la anulación de uno u otro de los términos, concibiéndolos excluyentes. De esa manera, se ha restado complejidad y especificidad al problema de las relaciones históricas entre los modos de movilización de la sociedad. Por una parte, ciertas lecturas economicistas del materialismo histórico reducen toda novedad en los procesos sociales contemporáneos e insisten con la exclusividad del movimiento obrero industrial como sujeto de cambio. Por otra parte, las teorías de raigambre funcional-estructuralista resaltan de tal modo la novedad de los movimientos sociales que terminan por perder de vista cualquier vínculo con las experiencias pasadas, con el problema del poder y con el cambio social.

Con la intención de esbozar posibles elementos de continuidad y ruptura entre formas de luchas pasadas y presentes en un escenario local, en el presente trabajo se indagan la génesis y el desarrollo del Movimiento de Trabajadores Desocupados (en adelante MTD) en la provincia del Chaco, haciendo referencia puntualmente a los diversos casos de "toma" de tierras que aparecen a lo largo del proceso.

Las "tomas", por su carácter instrumental, se han vinculado de distintas maneras con los procesos de movilización social a lo largo de la historia. En nuestro caso, se analizan sus múltiples manifestaciones en relación con los sectores desempleados de la clase obrera, tanto en episodios anteriores como posteriores a su conformación definitiva en un "movimiento", entendido este como expresión de la conformación de una fuerza social. Para ello se realiza una reconstrucción histórica de tres casos en contextos y momentos diferentes: el asentamiento de Santa Catalina, ocurrido entre 1950 y 1969, y posteriormente ampliado por el MTD Zona Norte; el asentamiento "Mate Cosido", impulsado en 2002 por el Movimiento Territorial de Liberación (MTL), y las ocupaciones del MTD 17 de Julio, consolidadas en 2006.

La información utilizada para elaborar el trabajo proviene de entrevistas realizadas a los principales referentes de las organizaciones mencionadas, artículos periodísticos publicados en la prensa local y bibliografía específica sobre la temática.

\section{Movimientos sociales $\mathrm{y} / \mathrm{o}$ lucha de clases}

El denominado "movimiento piquetero" o Movimiento de Trabajadores Desocupados $^{1}$ es concebido como un fenómeno novedoso en la Argentina

\footnotetext{
${ }^{1}$ La denominación "Movimiento de Trabajadores Desocupados" (MTD) es utilizada algunas veces para referir al conjunto del movimiento piquetero y otras veces para referir a una organización en particular. En
} 
contemporánea y ha sido objeto de numerosos estudios en el ámbito de las Ciencias Sociales durante los últimos años. Gran parte de los trabajos de investigación centra su atención en la diferencia entre los movimientos sociales del pasado (cuyo paradigma eran las luchas de los obreros industriales) y los "nuevos movimientos sociales", categoría que engloba a una diversidad de grupos de protesta que cobraron mayor visibilidad en Europa durante la segunda mitad del siglo XX: desocupados, ecologistas, estudiantes y feministas, entre otros.

Las escuelas norteamericana y europea que construyen sus marcos conceptuales en torno de la categoría de "movimiento social" ponen el acento, respectivamente, en la racionalidad estratégico-instrumental de la acción colectiva para administrar los recursos de la organización, o bien en la confrontación simbólica cuyo objetivo central es la construcción de una identidad que permita la satisfacción de las expectativas (Galafassi, 2006). De esta manera, sobre la base de un fuerte individualismo metodológico, se elude la lectura ideológico-política de las luchas populares y se deja de lado el conflicto por el poder y el cambio social.

En el mismo marco se incluye la impugnación por parte de las mencionadas escuelas de los conceptos asociados al materialismo histórico (clase social, lucha de clases, ejército industrial de reserva), por su supuesta incapacidad para dar cuenta de las nuevas características de los movimientos sociales, así como la desvinculación tajante entre formas actuales y pasadas de lucha popular. En consecuencia, no solo se concluye la necesidad de categorías teóricas enteramente nuevas para analizar los fenómenos contemporáneos, sino que también se los concibe de manera aislada con respecto de sus antecedentes históricos.

"... al identificar las teóricas sociológicas neo-funcionalistas y pos-estructuralistas de los movimientos sociales a los 'nuevos' como algo que emerge por primera vez y que se separa de los 'viejos', se está promoviendo una inmovilización del proceso histórico (...) Esto equivale a negar la dinámica procesual, ya que lo nuevo sería un emergente sin conexión con lo viejo" (Galafassi, 2006: 49).

Por otra parte, los estudios que sostienen la pertinencia de las categorías del materialismo histórico para el análisis de las luchas sociales de la actualidad destacan la importancia de indagar las continuidades y rupturas entre formas de lucha, entendiéndolas como acumulación de experiencias por parte de una fuerza social (Benclowicz, 2011; Iñigo Carrera, 2008; Izaguirre \& Aristizábal, 1988; Klachko, 1999; Marín, 2007; Oviedo, 2004). En este sentido, se entiende que las fuerzas sociales se constituyen en los enfrentamientos y suponen alianzas entre distintas fracciones de

este último caso se agrega una denominación que diferencia a esa organización de las demás, como por ejemplo: Movimiento de Trabajadores Desocupados 17 de Julio. 
clases, cada una con un conjunto de experiencias, prácticas y conocimientos históricamente acumulados (Iñigo Carrera, 2008).

Desde esta perspectiva no se niegan los cambios históricos y su influencia en la lucha popular, sino que se concibe a los sujetos emergentes como expresiones de los nuevos ámbitos de la clásica contradicción entre capital y trabajo. "Lo que no cambia sustancialmente es la base estructural de las relaciones de explotación y dominación de toda sociedad capitalista, aunque hayan cambiado las formas que adopta el proceso de explotación" (Galafassi, 2006: 50).

Sobre el MTD, particularmente, los teóricos de esta perspectiva señalan el papel decisivo de un experimentado activismo obrero que en los 90 pasa a engrosar las filas del amplio espectro que constituye el ejército industrial de reserva. De este modo, las conquistas organizativas y programáticas adquiridas en el curso de sus experiencias de lucha pasan a formar parte de la memoria colectiva de la clase y se renuevan bajo las condiciones del contexto. "Es natural, entonces, que el movimiento piquetero renovara, bajo nuevas condiciones, la tradición histórica de la clase obrera argentina" (Oviedo, 2004: 4). Al mismo tiempo, la conformación del MTD permitió incluir en el movimiento obrero a un sector que había permanecido tradicionalmente al margen de las luchas: precisamente, al sector de los trabajadores desempleados y subempleados. "Su novedad reside en el hecho de que se constituyó como un nuevo ámbito de sociabilidad en el que participaron nuevos contingentes de la clase obrera, conjugando la creatividad del presente con la experiencia del pasado". (Benclowicz, 2011: 99).

Es desde esta perspectiva teórico-metodológica que encaramos el estudio pormenorizado de una manifestación local del MTD, en nuestro caso referido a tres organizaciones de la provincia del Chaco. Con ello, intentamos poner en evidencia las líneas de continuidad entre las expresiones del "movimiento piquetero" y otras experiencias históricas de movilización social. En particular, una de las formas de lucha que vincula ambos términos de la relación es la ocupación de tierras (en adelante "toma") y su posterior utilización para la instalación de viviendas o de emprendimientos productivos.

La toma es una medida de lucha de carácter instrumental, en tanto señala un momento táctico en el proceso de formación y desarrollo de una fuerza social (Izaguirre \& Aristizábal, 1988; Marín, 2007). Sus significados provienen de la mediación social que los implemente. Por lo tanto, al igual que el MTD, posibilita incorporar en la alianza social a aquellos sectores de la clase obrera que no mantienen una vinculación permanente con el proceso productivo.

\section{MTD Zona Norte: transformaciones de una forma de acción directa}

El Movimiento de Trabajadores Desocupados "Zona Norte", surgido en Resistencia (Chaco) en el año 2000, se encuentra vinculado con dos procesos de tomas, 
correspondientes a momentos y contextos diferentes: uno, en los comienzos de la organización territorial que precedió a la conformación del MTD y otro, posterior a su consolidación, con objetivos y características distintas.

La primera toma coincidió con el momento mismo de formación del barrio Santa Catalina, la principal base territorial del futuro movimiento. Entre 1950 y 1969 se produjo el asentamiento espontáneo y progresivo de familias expulsadas de las zonas rurales del interior del Chaco y de Corrientes en terrenos baldíos de la periferia de Resistencia. Pertenecientes a una fábrica de tanino abandonada, y rodeada de sus antiguas lagunas de desagüe, los terrenos carecían de valor inmobiliario por encontrarse en un área inundable. El asentamiento adoptó la forma típica de "villa miseria", uno de los recursos históricamente utilizados por los excluidos de las grandes ciudades latinoamericanas:

“... la villa ha sido la forma más tradicional del hábitat popular urbano de la región y ha sido el resultado de lentas y no planificadas ocupaciones de tierras urbanas y suburbanas intersticiales de muy baja calidad (anegadizas, pantanosas, etc.)" (Romagnoli, 2004: 12).

El avance de la toma fue paralelo al de la organización territorial del barrio. En 1967 se constituyó formalmente la Comisión Vecinal de Santa Catalina, encabezada por militantes de la Juventud Peronista (JP). Desde su nacimiento, la comisión mantendría una relación con el Partido Justicialista caracterizada por el claro apoyo en tiempos electorales y por la utilización de medidas de acción directa ante el incumplimiento de sus reclamos. ${ }^{2}$ Sus principales actividades se vincularon inicialmente con la realización de protestas para solucionar la marcada precariedad habitacional y de servicios básicos del barrio, así como la extendida desocupación de sus habitantes.

La comisión obtuvo su primer logro importante en 1975, después de una movilización y "toma" de la Casa de Gobierno de la provincia. Aunque los vecinos reclamaban originalmente solo la expropiación de los terrenos del barrio, lograron por parte de la Subsecretaría de Vivienda la firma de un convenio con la Universidad del Nordeste para la colaboración técnica en un Plan de Consolidación. Este incluía desde la mensura y regularización de los terrenos, hasta la construcción de módulos nucleares de viviendas para las 217 familias del barrio, mediante la participación conjunta de vecinos y técnicos (Pelli \& Matta de Moreschi, 1981).

El Plan de Consolidación fue interrumpido en 1977 por los funcionarios de la Subsecretaría de Vivienda que habían sido nombrados por el gobierno de facto. Desde entonces, la Comisión Vecinal de Santa Catalina subsistió solo a título nominal hasta

\footnotetext{
2 Entrevista con el líder histórico de la Comisión Vecinal de Santa Catalina y dirigente del Movimiento de Trabajadores Desocupados Zona Norte. Resistencia, 17 de diciembre de 2009.
} 
casi la finalización de la dictadura militar. Su accionar quedó notablemente restringido y algunos de sus miembros, entre ellos el líder histórico de la comisión, estuvieron detenidos en la Brigada de Investigaciones de Resistencia.

Una nueva etapa de movilización comenzó en 1982, cuando la comisión encabezó las medidas de fuerza para reclamar la reparación de los daños sufridos por el barrio en las inundaciones de ese año. En 1984 se conformó desde ese espacio la Cooperativa de Trabajo Asociado y Consumo Juan Martín de Pueyrredón, lo cual permitió a los vecinos trabajar en la instalación de redes de infraestructura básica, viviendas y equipamiento comunitario hasta fines de los 90. A lo largo de esos años, la población de Santa Catalina creció vertiginosamente como producto del crecimiento vegetativo natural de las familias originales del asentamiento. Sus habitantes pudieron resistir la presión inmobiliaria, generada por la revalorización de la zona, y permanecieron en el barrio gracias a su alto grado de organización comunitaria (Romagnoli, 2004: 54-66).

En el año 2000 se registra una caída en el nivel de participación de los pobladores del barrio en la Comisión Vecinal, pero no así de su capacidad de autogestión. Dos factores parecen haber confluido en una redirección de los intereses del grupo que encabezaba la comisión hacia ámbitos de acción extrabarriales y a una ampliación de sus bases sociales: por un lado, los procesos organizativos de larga data habrían permitido una "toma de conciencia por parte de la comunidad de la gestión asociada", y por otro lado, los logros obtenidos por los pobladores en cuanto a equipamiento comunitario constituyeron "nexos de integración social entre el barrio y su entorno" (Romagnoli, 2004: 107-110). Es así que en la misma época y con el mismo líder histórico de la Comisión Vecinal surge el Movimiento de Trabajadores Desocupados Zona Norte, integrado por habitantes del barrio Santa Catalina y adyacentes (Villa Gonzalito, Atlántico Sur). La nueva organización centraría sus reclamos en la generación de puestos de trabajo y sería concebida, ya no como una asociación de vecinos, sino como un "movimiento político y social".

EI MTD Zona Norte, como una más de las formas organizativas adoptadas por la comunidad, no habría anulado las formas anteriores, sino que las habría incluido en un proyecto más amplio capaz de superar los límites barriales y las reivindicaciones materiales inmediatas. Esto se hace evidente en la descripción del uso que el movimiento da a la Cooperativa creada casi veinte años antes: "La cooperativa es una figura jurídica que puede captar trabajo. Pero es para todos, para compartir con el

\footnotetext{
${ }^{3}$ Entrevista con el líder histórico de la Comisión Vecinal de Santa Catalina y dirigente del Movimiento de Trabajadores Desocupados Zona Norte. Resistencia, 17 de diciembre de 2009. Es llamativa la similitud de la trayectoria histórica del MTD Zona Norte con la Federación de Tierra y Vivienda (FTV), una de las organizaciones piqueteras más numerosas del país, también vinculada con el peronismo (Svampa \& Pereyra, 2003: 44).
} 
movimiento, para compartir con los otros. Gente de otro lado, no solamente de la comunidad". ${ }^{4}$

El movimiento adquirió definitivamente visibilidad pública a mediados de $2001^{5}$, cuando participó, junto con otras organizaciones de desocupados, de una movilización que derivó en la suspensión de un acto en el cual el entonces presidente Fernando De la Rúa pretendía inaugurar el Plan Jefes y Jefas de Hogar (Pratesi, 2009). El "piquete" de calles y rutas, así como las movilizaciones hacia la Casa de Gobierno de la provincia serían desde entonces las principales metodologías utilizadas por el MTD Zona Norte para reclamar asistencia social y puestos de trabajo. Este objetivo se concretaría mediante el acceso a planes de empleo, en un primer momento, y posteriormente a través del ingreso como personal permanente en dependencias estatales (Ministerio de Salud, Ministerio de Desarrollo Social, Instituto de Vivienda, escuelas) o como personal contratado en obras públicas.

Asimismo, en un proceso que se continúa hasta el presente, el movimiento comenzó a impulsar el desarrollo de emprendimientos productivos en barrios no adyacentes a Santa Catalina y en el interior de la provincia. Con esta intención, integrantes del MTD Zona Norte ocuparon terrenos baldíos cercanos al barrio Santa Catalina en el año 2002. Mediante la cooperativa se gestionaron proyectos productivos (agricultura y cría de cerdos) con asistencia del Estado nacional. No obstante, los mecanismos de ocupación fueron completamente diferentes de los utilizados en la conformación del barrio, unos cuarenta años antes. Entre las características novedosas del proceso, al igual que otros casos en la ciudad, caben señalarse las siguientes: "Son ocupaciones masivas que se producen repentinamente, en terrenos privados, donde las familias ocupantes se organizan en el espacio en forma regular distribuyendo el terreno ocupado en lotes de dimensiones aproximadamente iguales para todas las familias ocupantes" (Benítez, 2000). La nueva forma de los asentamientos supone un nivel de organización de los participantes con múltiples fines (instalación, resistencia al desalojo, obtención de beneficios comunes) y en diversos niveles (interno, entre los vecinos de un mismo asentamiento, y externo, en vinculación con otros asentamientos $u$ otras organizaciones), que se explica por la pertenencia de los ocupantes a grupos organizados previamente existentes.

Por último, cabe destacar el planteo por parte del MTD Zona Norte de un programa político de reforma del Estado, en cuyo marco se incluyen tanto la participación del proceso de luchas contra las medidas implementadas durante la presidencia de

\footnotetext{
4 Ídem.

${ }^{5} \mathrm{Si}$ bien los testimonios dan cuenta de movilizaciones anteriores, los periódicos locales registran por primera vez el nombre del MTD Zona Norte en una movilización del 5 de junio de 2001, en ocasión de la visita del presidente De la Rúa a la provincia del Chaco. Cfr. "Momentos de pánico en el Domo del Centenario". Norte. 06/06/2001. Locales, p. 4.
} 
Fernando De la Rúa hasta su renuncia, como el apoyo al gobierno en funciones desde 2007, tanto a nivel provincial como nacional.

\section{MTL: las ventajas de una organización preexistente}

El Movimiento Territorial de Liberación (MTL) es una organización que surge en la provincia del Chaco en el año 2002, como delegación local de la organización nacional del mismo nombre, vinculada con el Partido Comunista Argentino (PCA). Sus principales cuadros dirigentes provenían de este partido, aunque habían atravesado procesos de lucha política en ámbitos diversos y ya habían conformado su propia organización de desocupados, junto con sectores extrapartidarios.

A fines de los 90 los militantes del PCA en la provincia del Chaco construyeron sus bases sociales en la militancia sindical, principalmente en los sectores docentes y de trabajadores de la construcción. Ambos confluyeron en el apoyo a los miles de obreros que quedaron desocupados y sin representación gremial, impulsando la formación del primer movimiento de trabajadores desocupados chaqueño, el MTD General San Martín. Quien más tarde sería la principal dirigente del MTL se incorporó a este proceso después de haber participado en la organización del XIII Encuentro Nacional de Mujeres, realizado en la ciudad de Resistencia (Chaco) en 1998. ${ }^{6}$ Dicho evento, actualmente constituido como una referencia a nivel nacional, había comenzado a realizarse en 1986 y era concebido como un ámbito "autónomo, autoconvocado, democrático, pluralista y horizontal" ${ }^{77}$, cuyo objetivo era analizar las luchas y las formas de discriminación respecto de las mujeres. La convocatoria reunió a 10 mil mujeres de todo el país, quienes elaboraron un documento en el cual reclamaban "más espacios en el poder". ${ }^{8}$

EI MTD General San Martín protagonizó importantes luchas en la provincia desde su fundación, en julio de 1999, hasta su división. En noviembre de 2000 sufrió una primera escisión del grupo que más tarde conformó el MTD 17 de Julio. Ante esto, los militantes del PCA decidieron sostener el movimiento y comenzar un proceso de reorganización, esta vez con un mayor respaldo de su partido. No obstante, poco después comenzaron a expresarse nuevas diferencias en el interior del MTD General San Martín.

Uno de los grupos, con presencia mayoritaria en la ciudad de Barranqueras, pasó a denominarse MTD General San Martín de Barranqueras. Los líderes se habían alejado del Partido Comunista y se encontraban entablando relaciones con el Partido Obrero

\footnotetext{
${ }^{6}$ Entrevista con dirigentes del Movimiento Territorial de Liberación. Resistencia, 10 de abril de 2009.

${ }^{7}$ Resistencia las espera! Invitación de la Comisión Organizadora al XIII Encuentro Nacional de Mujeres.

8 "Diez mil mujeres se reúnen en Resistencia”. El Diario. 24/12/1998. Anuario, p. 27.
} 
(PO). Un año más tarde conformarían, con la misma base social, el Polo Obrero, una organización de desocupados alineada a nivel nacional con el PO.

La otra parte del MTD General San Martín, liderado por quienes permanecían en el Partido Comunista (PC) y cuya base social mayoritaria se hallaba en los barrios de Resistencia, estableció lazos con la dirección nacional del MTL en 2002, al cual se incorporaron ese mismo año (Poli, 2007: 25-34). La primera aparición pública del MTL en el Chaco se produjo el 26 de junio de 2002, en el marco de las "Jornadas de lucha" impulsadas por la Asamblea Nacional Piquetera contra la política económica del gobierno nacional. ${ }^{9}$

En agosto de 2002, unas doscientas familias del MTL ocuparon terrenos particulares en un área intersticial de los suburbios de Resistencia, con la intención de construir un complejo habitacional. Al igual que el MTD Zona Norte por la misma época, el MTL había organizado la ocupación y planificado el uso de los espacios dentro del futuro barrio. El asentamiento fue nombrado "Mate Cosido", en referencia al alias con el que fue conocido uno de los más famosos bandidos rurales de la zona, Segundo David Peralta (Benítez, 2008). Después de resistir un intento de desalojo por la fuerza policial, los ocupantes aceptaron la propuesta del gobierno provincial para la relocalización. En el nuevo predio, la construcción de las viviendas por sus mismos habitantes se inició en enero de 2003. Actualmente residen allí unas doscientas familias pertenecientes al MTL, que continúa trabajando en la ampliación del barrio. Sus integrantes se encuentran llevando a cabo la construcción de un complejo educativo gestionado por la propia comunidad.

\section{MTD 17 de Julio: la toma de tierras para producir}

El MTL, el Polo Obrero y el MTD 17 de Julio comparten el mismo proceso de formación mencionado. No obstante, estas dos últimas organizaciones en particular estuvieron precedidas por una importante trayectoria de militancia sindical entre los obreros de la construcción.

A mediados de los 80, quienes serían los principales dirigentes del Polo Obrero y del MTD 17 de Julio trabajaban para empresas constructoras de la provincia del Chaco. En el marco de las huelgas nacionales contra el gobierno de Alfonsín conformaron una línea interna dentro de la UOCRA, denominada "Agrupación naranja violeta". Desde allí impulsaban la formación política de los trabajadores, la elección de delegados en asambleas y la realización de medidas de acción directa en las protestas. Con el aumento de los índices de desocupación en los 90, su militancia se volcó hacia el sector de trabajadores desocupados del rubro, desarrollando una base territorial en los

\footnotetext{
9 "El bloque piquetero provincial cortó la ruta 11". La Voz del Chaco. 27/06/2002. Locales, p. 4. "Siete piquetes en la ruta $11^{\prime \prime}$. Norte. 27/06/2002. Locales, p. 32.
} 
barrios que habitaban. Los reclamos se centraron en el pago de los seguros de desempleo, la obtención de mejoras de infraestructura barrial y el mejoramiento de viviendas mediante autoconstrucción. En ese marco se produjo la inundación de 1998, que afectó numerosos barrios de Resistencia y Barranqueras.

Los vecinos inundados de las localidades metropolitanas organizaron una masiva movilización hasta la Casa de Gobierno provincial para reclamar la reparación de las viviendas inundadas y la generación de puestos de trabajo. La obtención de soluciones parciales impulsó a un grupo de ellos a seguir manifestándose mediante cortes de calle. Estos confluyeron con militantes comunistas, socialistas y peronistas en julio de 1999, conformando el MTD General San Martín.

En noviembre de 2000 un grupo se separó del movimiento original y formó el Frente de Liberación Nacional y Social. En enero de 2001 el mismo grupo adoptó el nombre definitivo de MTD 17 de Julio, en conmemoración de la primera gran represión resistida por los desocupados chaqueños un año antes. Merced a la repercusión mediática de la detención de su principal dirigente en febrero de 2001 y a su participación en las Asambleas Nacionales Piqueteras en el marco de la creciente protesta social contra el gobierno nacional, este movimiento ocupó un lugar importante entre las agrupaciones denominadas "autónomas" de todo el país, a pesar de no encontrarse vinculada con ninguna organización nacional. Durante los años siguientes estableció sucesivas alianzas con distintas agrupaciones del mismo tipo: el Movimiento Teresa Rodríguez, la Coordinadora de Trabajadores Desocupados Aníbal Verón y el Movimiento Independiente de Jubilados y Desocupados. Ninguna de ellas prosperó durante mucho tiempo.

Después de participar en la mencionada coalición electoral del año 2003, el MTD 17 de Julio comenzó a concebir el desarrollo de proyectos productivos como una alternativa a los subsidios por desempleo, capaz de generar independencia económica. En un contexto marcado por la implementación masiva de planes sociales en el Chaco y la fragmentación de las organizaciones de desocupados ante el gobierno nacional de Néstor Kirchner, el movimiento proyectó, en principio, la realización de emprendimientos agropecuarios en zonas rurales del interior de la provincia. Sobre la base de pequeñas experiencias de algunos de sus integrantes, se reclamó la entrega de herramientas, semillas y de tierra para 600 familias, sin obtener respuestas del gobierno provincial. $^{10}$

El desarrollo de los emprendimientos productivos empezó a concretarse recién a principios de 2005. La ocupación durante un mes del edificio de la Gerencia de Empleo

10 "Desocupados quieren 50 hectáreas de tierra y el aumento a $\$ 300$ de los planes sociales". Norte. 31/10/2003. Información General, p. 44 
en el Chaco permitió al MTD 17 de Julio obtener la posibilidad de presentar proyectos para la cría de animales de granja, apicultura y ladrillería. ${ }^{11}$

Los proyectos productivos significaban para el movimiento la posibilidad de dejar de depender del Estado y obtener sus recursos mediante el trabajo propio. A diferencia de los planes sociales, eran concebidos como un avance en la independencia económica y, en consecuencia, en la autonomía política que aparece en el imaginario de la organización como uno de los objetivos principales. ${ }^{12}$

La asistencia del Estado nacional implicaba el otorgamiento de herramientas, insumos y capacitación técnica, pero no así de terrenos para la realización de los emprendimientos. Por lo tanto, el movimiento decidió reclamarlas al gobierno provincial. A principios de 2006, se logró el otorgamiento de tierras fiscales en dos localidades del interior por parte del Instituto de Colonización de la provincia. Sin embargo, la intención original de desarrollar los emprendimientos en zonas rurales quedó trunca por el rechazo de los propietarios de campos cercanos a los otorgados al movimiento. En el momento de tomar posesión de las tierras hubo amenazas y enfrentamientos con armas de fuego, ya que los vecinos se oponían a la convivencia con los "piqueteros". Ante la magnitud de los hechos, en los cuales dos integrantes resultaron heridos de bala, la organización decidió ocupar tierras fiscales en la periferia del Gran Resistencia y construir por sí mismos las instalaciones que necesitaban. En abril de 2006, luego de lograr la entrega de los animales de granja, integrantes del MTD 17 de Julio ocuparon unas 10 hectáreas en la ribera del río Paraná con la intención de desarrollar 20 proyectos productivos de cría de cerdos y pollos. ${ }^{13}$

Una vez instalados los emprendimientos, algunos en tierras ocupadas y otros en viviendas particulares, su desarrollo posterior no estuvo exento de conflictos. Los incumplimientos de los gobiernos nacional y provincial en materia de capacitación e insumos constituyeron motivos centrales de la protesta del movimiento durante los años siguientes. A pesar de todo, la posibilidad de generar puestos de trabajo propios pasó a formar parte desde ese momento de las actividades del MTD 17 de Julio hasta la actualidad.

\section{Consideraciones finales}

En primera instancia, la descripción del desarrollo histórico de tres organizaciones pertenecientes al Movimiento de Trabajadores Desocupados del Chaco evidencia una compleja red de relaciones con luchas sociales de épocas anteriores, no solo la toma de

\footnotetext{
11 "Piqueteros dialogaron con Gladis Soto". Norte. 31/12/2004. Locales, p. 17

12 Entrevista con un dirigente del MTD 17 de Julio. Resistencia, 14 de abril de 2008.

13 "Piqueteros ocuparon diez hectáreas en Antequera". Norte. 30/04/2006. Locales, p. 18.
} 
tierras. Ese cúmulo diverso de experiencias históricas se unifica progresivamente hacia una matriz común que expresa la conformación de una fuerza social.

La diversidad se manifiesta en los ámbitos propios de las instancias de organización que precedieron a la formación de los movimientos, principalmente la militancia vecinal y sindical. La similitud, en tanto, se observa principalmente en la pertenencia a una matriz que podríamos denominar "piquetera", por ser el núcleo característico de todos los "movimientos piqueteros": la organización territorial como base del reclamo por alternativas al desempleo mediante medidas de acción directa. Esta matriz reúne de un modo peculiar elementos provenientes de otros tipos y ámbitos de militancia social combinados en un contexto específico, como fue la década de los 90 en Argentina. Por esta razón, es imposible concebir a las organizaciones de desocupados como un fenómeno absolutamente novedoso. Más bien, podemos conjeturar que a lo largo del proceso se produce la conformación de una fuerza social que apela a la experiencia histórica de la clase en sus formas de organización y acción directa (principalmente el "piquete" y la toma), al tiempo que incorpora a la lucha a los contingentes desempleados de la clase.

En el caso particular abordado en este trabajo, probablemente el rasgo paradigmático en cuanto a líneas de continuidad entre las tradiciones de lucha y el Movimiento de Trabajadores Desocupados sea la toma de tierras. Las tres organizaciones analizadas protagonizaron este tipo de hechos en algún momento de su desarrollo, aunque en cada caso con características específicas.

Las bases sociales del MTD Zona Norte, aun antes de su conformación como organización, provienen de un proceso de tomas que configura desde el inicio su espacio habitacional. No obstante, las características de este proceso responden a un formato "viejo" que se corresponde con la construcción de "villas miseria" durante las décadas de 1970 y 1980 en nuestro país: la ocupación de tierras periféricas y sin valor inmobiliario, mediante un asentamiento lento (a lo largo de décadas) y no planificado, protagonizado por migrantes rurales. La organización, tanto del espacio mediante el operativo experimental, como de los pobladores en la Comisión Vecinal, es posterior a la toma.

Décadas más tarde, el cambio en la configuración organizativa de los pobladores hacia una organización específica de desocupados transformó también la metodología de ocupación. La nueva toma, producida en 2002, fue planificada y llevada a cabo por el movimiento, en un breve período, con una organización espacial determinada y protagonizada por los nuevos miembros de las familias habitantes del barrio original. Esta dinámica parece coincidir con las tomas realizadas por el MTL en el mismo año y por el MTD 17 de Julio algunos años después.

En particular, la toma y posterior construcción del barrio "Mate Cosido", aunque con un nuevo formato, tuvo el mismo objetivo que las ocupaciones "viejas": la obtención de 
un espacio habitacional. Asimismo, la preexistencia de una organización permitió ampliar los logros de esa toma mediante la resistencia al desalojo, la negociación con instancias de gobierno, la autoconstrucción de viviendas y la proyección de equipamiento comunitario (específicamente, un centro educativo).

Por último, la toma protagonizada por el MTD 17 de Julio innovó en formato y también en objetivos. A las características anteriormente mencionadas se agregó la utilización de las tierras para el desarrollo de emprendimientos productivos como parte de un imaginario que concebía a la producción rural como herramienta de independencia económica y política.

En definitiva, la novedad relativa del "movimiento piquetero" en la Argentina reciente no reside tanto en su desvinculación absoluta de las formas de lucha popular precedentes, como en su particular modo de articulación entre experiencias históricas provenientes de ámbitos de militancia diversos. Ese fenómeno sería expresión de la formación de una fuerza social de carácter obrero a nivel provincial, en el marco de un ascenso de las luchas populares en todo el país. Tanto la trayectoria de los principales referentes de cada movimiento, como la utilización renovada de una forma tradicional de acción directa, la toma, permiten dar cuenta de ello. 


\section{Bibliografía y fuentes}

Benclowicz, J. D. (2011). Repensando los orígenes del movimiento piquetero. Miseria y experiencias de lucha antes de las contrarreformas de la década de 1990 en el norte argentino. Latin American Research Review. The journal of the Latin American Studies Association, 46 (2), 79-103.

Benítez, A. (2000). Ocupaciones de tierra, Movimientos sociales y Construcción de Identidad. Un análisis de los Movimientos de Trabajadores Desocupados y Ocupantes de Tierra en Resistencia, Chaco. Topos y Tropos, 4. Disponible en URL: http://www.toposytropos.com.ar/N4/tesis/benitez.htm

----------(2008). Intervenciones estatales en materia habitacional en asentamientos en Resistencia, Chaco. Área Digital, 10. Disponible en URL: http://arq.unne.edu.ar/sitioanterior/institucional/publicaciones/areadigital/area10/doc umentos/pdf/benitez.pdf

Galafassi, G. (2006). Cuando el árbol no deja ver el bosque. Neofuncionalismo y posmodernidad en los estudios sobre movimientos sociales. Revista Theomai, 14, 37 58.

Iñigo Carrera, N. (2008). Algunos instrumentos para el análisis de las luchas populares en la llamada Historia Reciente. En López Maya, M.; Iñigo Carrera, N. y Calveiro, P. (eds.) Luchas contrahegemónicas y cambios políticos recientes de América Latina (pp. 77-94). Buenos Aires: CLACSO.

Izaguirre, I. y Aristizábal, Z. (1988). Las tomas de tierras en la zona sur del Gran Buenos Aires. Un ejercicio de formación de poder en el campo popular. Buenos Aires: Centro Editor de América Latina.

Klachko, P. (1999). Cutral Có y Plaza Huincul. El primer corte de ruta (del 20 al 26 de junio de 1996). Cronología e hipótesis. PIMSA. Publicación del Programa de Investigación sobre el Movimiento de la Sociedad Argentina. Documentos y comunicaciones 1999, 3, 121-154.

Marín, J. C. (2007). El ocaso de una ilusión. Chile 1967/1973. Buenos Aires: Colectivo Ediciones / PICASO / INEDH.

Oviedo, L. (2004). Una historia del movimiento piquetero. De las primeras Coordinadoras al Argentinazo. Buenos Aires: Rumbos.

Pelli, V. S. y Matta de Moreschi, S. (1981). Consolidación de barrios marginales: teoría y ejecución de una experiencia en el Barrio Santa Catalina. Resistencia: Departamento de Diseño del Equipamiento Residencial, Facultad de Arquitectura y Urbanismo, Universidad Nacional del Nordeste.

Poli, C. (2007). Movimiento Territorial de Liberación. Su historia. Piquetes, organización, poder popular. Buenos Aires: Centro Cultural de la Cooperación Floreal Gorini. 
Pratesi, A. (2009). Identidades de la pobreza. Honestos, chorros y piqueteros. Resistencia: El Apagón.

Pratesi, A. y Rozé, J. (2005). Movimiento de desocupados en Resistencia, Chaco. Fracciones de izquierda para una política hegemónica. En: Pratesi, Ana y Rozé, Jorge (comps.) Conflictos centrales en la periferia de la globalización (205-223). Resistencia: LibrosEnRed - Fundación IdEAS.

Romagnoli, V. (2004). Casos experimentales de resolución habitacional. Hipótesis iniciales - situación actual: Barrio Santa Catalina. Informe Final Beca de Iniciación a la Investigación. Resistencia: Instituto de Investigación y Desarrollo en Vivienda, Facultad de Arquitectura y Urbanismo, Universidad Nacional del Nordeste. (Inédito).

Svampa, M. y Pereyra, S. (2003). Entre la ruta y el barrio. La experiencia de las organizaciones piqueteras. Buenos Aires: Biblos.

\section{Prensa}

Artículos periodísticos publicados por los diarios locales Norte, El Diario y La Voz del Chaco entre 1998 y 2006.

\section{Entrevistas}

Entrevista con un dirigente del MTD 17 de Julio. Resistencia, 14 de abril de 2008.

Entrevista con dirigentes del Movimiento Territorial de Liberación. Resistencia, 10 de abril de 2009.

Entrevista con el líder histórico de la Comisión Vecinal de Santa Catalina y dirigente del Movimiento de Trabajadores Desocupados Zona Norte. Resistencia, 17 de diciembre de 2009. 